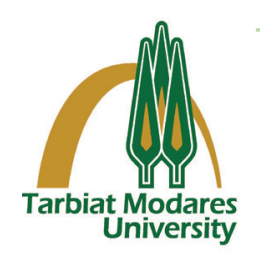

\title{
A Case study of Imported Plasmodium falciparum Infection in Bandar-e-Kong in Southern Iran
}

\section{A R T I C L E I N F O}

\section{Article Type}

Case Report

\section{Authors}

Zohreh Fakhrieh-Kashan, $P h D^{1}$ Marziyeh Yazdanpanah, $M S c^{2}$ Jafar Hatami Gurbandi, $M S c^{3}$ Sareh Bagheri-Josheghani, $M S C^{4}$ Maryam Arfaatabar, $P h D^{5}$ Maryam Ghobahi, $M S c^{2 *}$

\section{A B S T R A C T}

Backgrounds: Plasmodium species are the causative agents of malaria, which is considered as a mosquito-borne infectious disease transmitted by anopheles mosquitoes. It is endemic in the southern and south-eastern areas of the country. This study aimed to report a case of imported malaria infection in a Pakistani man in Bandare Kong located in the central region of Bandar Lengeh County in Hormozgan province, Iran.

Case presentation: A Pakistani male fisherman aged 21 years referred to a general practitioner in Bandare kong with symptoms such as a long-lasting fever that worsened every 2 days. He underwent a malaria rapid diagnostic test (RDT), and the result of which was positive. Therefore, for further malaria testing, he was dispatched to Shohada hospital in Bandar Lengeh. All the laboratory tests findings were in an acceptable range, except for direct bilirubin titer, which was higher than the normal range 0.3 $\mathrm{mg} / \mathrm{dL})(<0.2 \mathrm{mg} / \mathrm{dL}=$ normal). Blood smear test results also showed $P$. falciparum-associated malaria infection. According to the malaria therapy protocols followed in the hospital, 24 coartem pills at a dose of 4 pills per 12 hours were administered to the patient for 3 days, and then primaquine was administered simultaneously after three days, resulting in a rapid reduction in the parasitic load so that it could not be detected after 2 days.

Conclusion: In recent decades, most malaria patients diagnosed in Iran have been infected cases entered Iran from neighboring countries where malaria is endemic. Therefore, early diagnosis and therapy of imported malaria cases in endemic areas of Iran is essential and makes it possible to implement malaria preventive and control measures.

\section{Keywords: Plasmodium falciparum, Imported malaria, RDT, Iran}

\section{CITATION LINKS}

[1] Schapira AM, Zaim M, Raeisi A, Ranjbar M, Kolifarhood G, Nikpour F, et al. History of the successful struggle against malaria in the Islamic Republic of Iran: From the earliest records to imminent elimination. Tehran: Shayan Gostar Publication; 2018. [2] World Health Organization. World malaria report 2020: 20 years of global progress and challenges. Geneva: World Health Organization; 2020. [3] Vatandoost H, Raeisi A, Saghafipour A, Nikpour F, Nejati J. Malaria situation in Iran: 2002-2017. Malar J. 2019;18(1):1-7. [4] Hemami MR, Sari AA, Raeisi A, Vatandoost H, Majdzadeh R. Malaria elimination in Iran, importance and challenges. Int J Prev Med. 2013;4(1):88-94. [5] Sheikhzadeh K, Haghdoost AA, Bahrampour A, Raeisi A, Zolala $\mathrm{F}$, et al. Predicting malaria transmission risk in endemic areas of Iran: A multilevel modeling using climate and socioeconomic indicators. Iran Red Crescent Med J. 2017;19(4):e45132. [6] Kong X, Liu X, Tu H, Xu Y, Niu J, Wang Y, et al. Malaria control and prevention towards elimination: Data from an eleven-year surveillance in Shandong Province, China. Malar J. 2017;16(1):55. [7] Diallo MA, Badiane AS, Diongue K, Deme A, Lucchi NW, Gaye M, et al. Non-falciparum malaria in Dakar: A confirmed case of Plasmodium ovale wallikeri infection. Malar J. 2016;15(1):1-6. [8] World Health Organization. WHO malaria terminology. Geneva: World Health Organization; 2016. [9] UCSF Global Health Group. Eliminating malaria in Iran. Son Francisco: University of California; 2015. [10] Shahin A, Abdelbaky N, Emam S. A case of malignant malaria complicated by symmetrical peripheral gangrene. J Rheum Dis Treat. 2015;1:016.

\begin{abstract}
* Correspondence
Address: Infectious and Tropical Diseases Research Center Hormozgan Health Institute, Hormozgan University of Medical, Sciences Bandar Abbas, Iran maryamghobahi@gmail.com
\end{abstract}

\section{Article History}

Received: March 052021

Accepted: April 25,2021

Published: May 20,2021

Copyright(C) 2021, TMU Press. This open-access article is published under the terms of the Creative Commons Attribution-NonCommercial 4.0 International License which permits Share (copy and redistribute the material in any medium or format) and Adapt (remix, transform, and build upon the material) under the Attribution-NonCommercial terms. 


\section{Introduction}

Malaria is a mosquito-borne infectious disease caused by Plasmodium parasites, including P. falciparum, P. malariae, P. ovale, and $P$. vivax ${ }^{[1]}$. It remains as an important parasitic disease worldwide; malaria was estimated to cause 229 million infected cases and 409,000 deaths in 2019 worldwide ${ }^{[2]}$. Almost 15 countries, mostly among sub-Saharan African and Indian countries, are accounted for more than $80 \%$ of the global burden of malaria infection ${ }^{[2]}$. P. vivax and $P$. falciparum have been found to be endemic in the provinces of Sistan and Baluchestan as well as Hormozgan, located in the southeast and south of the country, respectively [3]. Since 2009, Iran has started a malaria pre-elimination program with the aim of complacently eliminating malaria by 2025 ${ }^{[4]}$. It should be noted that the total number of locally-transmitted malaria cases recorded in Iran decreased to less than 200 cases from 2015 to 2017, while the number of imported cases increased compared to the number of autochthonous and indigenous cases ${ }^{[1,5]}$. But the influx of malaria patients into Iran from neighboring eastern countries including Pakistan and Afghanistan, which are among malaria-endemic areas, is considered as a major challenge for implementing a malaria elimination program in Iran ${ }^{[1]}$. Microscopic observation of Giemsa-stained thick and thin blood smears is a method routinely used to identify malaria infection in endemic areas ${ }^{[3,6]}$. It is a simple and inexpensive method, but the detection limit highly depends on the microscopist's experience ${ }^{[6]}$. Recently, different types of molecular techniques have been used to detect this infection ${ }^{[7]}$; However, as they require some equipment and trained personnel, they are not commonly used ${ }^{[1,7]}$. The rapid diagnostic test (RDT) kits, including dedicated $P$. falciparum antigens horseradish peroxidase- 2 (HRP-2) and lactate dehydrogenase (LDH), are currently used in remote and epidemic areas as well as wherever malaria detection is difficult, to identify infected individuals or migrants; the application of this method could contribute to early detection of malaria cases, especially in rural areas ${ }^{[1]}$.

Usually in malaria-endemic areas, the infection is treated according to the national protocols designed for malaria therapy ${ }^{[8]}$.

Objectives: This study was conducted to report a case of malaria infection in a Pakistani fisherman in Bandar-e-kong located in Bandar Lengeh County, Hormozgan province, Iran.

\section{Case Presentation}

In September 2019, a Pakistani male fisherman aged 21 years referred to a general practitioner in Bandare kong with symptoms such as a long-lasting fever that worsened every 2 days; he traveled from Pakistan to Iran for the first time 14 days ago. Based on clinical symptoms, he was diagnosed with suspected malaria infection and underwent a malaria RDT, the result of which was positive. Then he was dispatched to Shohada hospital in Bandar Lengeh for further malaria testing. All the biochemical and CBC (complete blood count) tests findings were in an acceptable range, except for direct bilirubin titer, which was higher than the normal range $(0.3 \mathrm{mg} / \mathrm{dL})(<0.2 \mathrm{mg} / \mathrm{dL}=$ normal $)$ (Table 1).

Blood smear test results also showed a P. falciparum-associated malaria infection with a parasitic load of 5.2\% (Figure 1 and 2). Based on the malaria therapy protocols followed in the hospital, 24 coartem pills (artemether $20 \mathrm{mg} /$ lumefantrine $120 \mathrm{mg}$ ) at a dose of 4 pills per 12 hours were administered to the patient for 3 days, and then primaquin was administered simultaneously after three days, resulting in a rapid reduction in the parasitic load so that it could not be detected after 2 days. 
For further examination, blood samples were taken from the patient's relatives living with him and examined for malaria. The test results were negative.

Table 1) The results of biochemical and CBC tests performed on Pakistani fisherman in Bandar-e-kong, Iran

\begin{tabular}{lll}
\hline $\begin{array}{l}\text { Investiga- } \\
\text { tion }\end{array}$ & Result & Reference Range \\
\hline Complete Blood Count & \\
\hline PT & 12.2 & $12-14(\mathrm{sec})$ \\
\hline PTT & 32 sec & $24-35(\mathrm{sec})$ \\
\hline WBC & 5.5 & $3.5-11.5\left(10^{+3} / \mathrm{mL}\right)$ \\
\hline RBC & 5.28 & $3.9-5.8\left(10^{+3} / \mathrm{mL}\right)$ \\
\hline Hb & 13.8 & $12-17(\mathrm{~g} / \mathrm{dL})$ \\
\hline HCT & 41.9 & $36-51(\%)$ \\
\hline Plt & 203 & $150-450\left(10^{+3} /\right.$ \\
& & mL) \\
\hline Biochemistry & 133 & $<0.2(\mathrm{mg} / \mathrm{dL})$ \\
\hline Bilirubin D & 0.30 & $0.1-1.2(\mathrm{mg} / \mathrm{dL})$ \\
\hline Bilirubin T & 0.74 & $100-140(\mathrm{mg} / \mathrm{dL})$ \\
\hline BS & 137 & $8-22(\mathrm{mg} / \mathrm{dL})$ \\
\hline BUN & 13 & $0.8-1.3(\mathrm{mg} / \mathrm{dL})$ \\
\hline Cr & 1.23 & UP to $31(\mathrm{IU} / \mathrm{L})$ \\
\hline SGOT & 36 & $\mathrm{UP}$ to $41(\mathrm{IU} / \mathrm{L})$ \\
\hline SGPT & 23 & $80-306(\mathrm{IU} / \mathrm{L})$ \\
\hline ALK & 253 & $145(\mathrm{mg} / \mathrm{dL})$ \\
\hline Na & 3.8 & $(\mathrm{mg} / \mathrm{dL})$ \\
\hline K & & \\
\hline
\end{tabular}

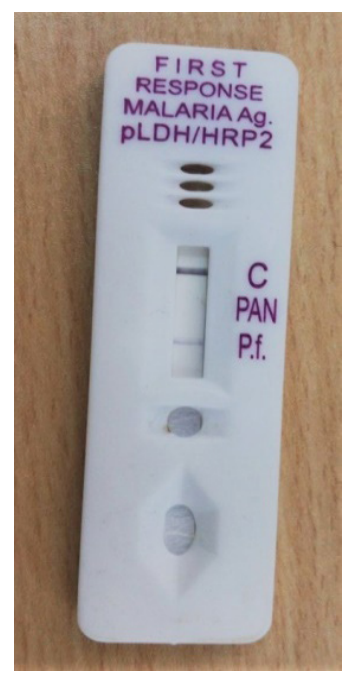

Figure 1) The result of rapid detection test kit (DH/ HRP2) used for the detection of malaria infection in Pakistani fisherman, Bandare kong, Iran

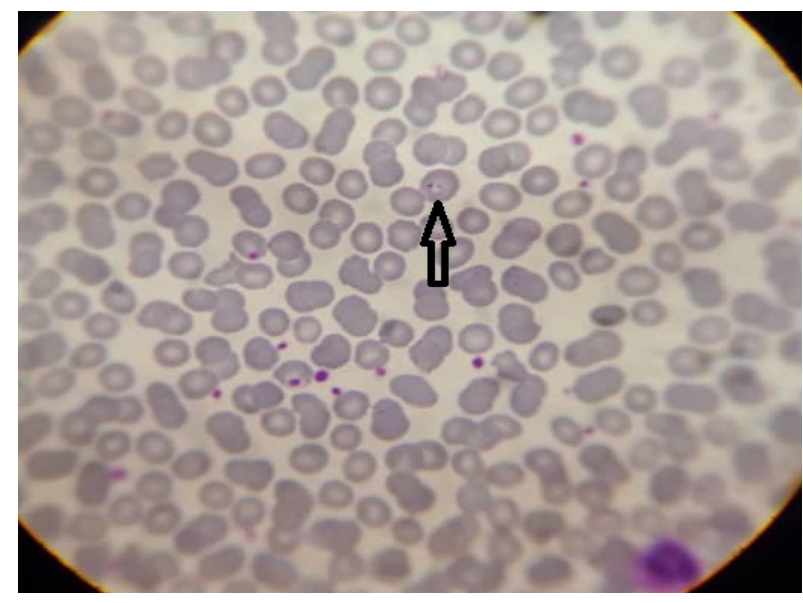

Figure 2) $P$. falciparum rings in red blood cells, Giemsa-stained thin blood smears with immersion lens (X100)

\section{Discussion}

Currently, malaria infection has been restricted to the southern and southeastern regions of the country with a very low incidence rate ${ }^{[3]}$. At the moment, a preeradication program is underway in Iran for malaria with WHO cooperation ${ }^{[4]}$. This study reported a case of imported malaria infection in a Pakistani fisherman infected with $P$. falciparum in an endemic area of Iran, while the malaria-elimination program was supposed to inhibit the transfer of $P$. falciparum by the end of 2015 and make Iran a country without malaria by $2025^{\left[{ }^{9]}\right.}$. Based on a recent study in Iran, the incidence rate of malaria decreased from 2009 to 2017 [3], whereas the number of imported malaria cases increased, which was mainly due to the influx of foreign immigrants and refugees from neighboring eastern countries, including Afghanistan and Pakistan [1, 3]. During 2002-2017, about 22209 Pakistani people with malaria infection entered Iran, most of whom were male and above 15 years of age ${ }^{[3]}$. The case reported in this study was similar to them.

Therefore, detection and evaluation of imported malaria cases are the most 
necessary measures to reach the target of malaria elimination in Iran. Hence, in remote and malaria-endemic areas and wherever the use of microscopic methods is not possible, the use of RDT could contribute to the early detection of malaria cases ${ }^{[1]}$. Also, the use of RDT was helpful in the early diagnosis of the patient studied. However, for definitive diagnosis, the standard examination of microscopic slides was performed based on the national malaria treatment guidelines. Feverishness, continuous pain in the head, dysphoria, abdominal pain, disgorging, bloodlessness, neutropenia, leukopenia, and hepatomegaly are among the common signs and symptoms associated with malaria infection ${ }^{[1]}$, whereas $P$. falciparum as the causative agent of malignant malaria could cause loss of consciousness, kidney dysfunction, hypotensive upset, and even loss of life ${ }^{[10]}$. Also, by early detection and suitable treatment of patients, complicated symptoms could be prevented. In the case reported in this study, the symptoms appeared in less than one week, while the parasitic load was only $5.2 \%$. Therefore, due to the early diagnosis of the disease, the patient was well treated, while he had only a little bilirubinemia, in addition to fever.

It should be noted that, malaria preelimination program is underway in Iran to passively and actively find suspected cases with symptoms associated with malaria such as fever ${ }^{[3]}$. Therefore, imported malaria cases are as controversial as indigenous cases and could hinder and delay the achievement of ultimate goal of complete malaria elimination.

\section{Conclusion}

Therefore, it is recommend that the surveillance system be promoted for early diagnosis and treatment of infected cases among foreigners, like Pakistanis and Afghans, using RDT and blood smear test due to the high level of worker population seasonal movements, especially in endemic areas of Iran.

Acknowledgments: The authors would like to thank all people who contributed to carry out this work, especially Shohada hospital laboratory employees in Bandar Lengeh for their technical help and Dr. Ranjbar for his valuable advice. They would also like to thank the patient for his efficient collaboration.

Ethical permission:This study was done according to Ethical guidelines of Hormozgan University of Medical Sciences.

Conflicts of interest: There are no conflicts of interest.

Authors' contribution: Conceptualization: All Authors,; Data curation and formal analysis: All Authors,; Investigation:All Authors,; Methodology and project administration: All Authors,; Supervision: MGH; Validation: MGH; Writing of original draft:All Authors; Writing, reviewing, and editing:All Authors.

Fundings: No Funding.

Consent to participate: Authors declare that all patient have signed the participation form.

\section{Reference}

1. Schapira AM, Zaim M, Raeisi A, Ranjbar M, Kolifarhood G, Nikpour F, et al. History of the successful struggle against malaria in the Islamic Republic of Iran: From the earliest records to imminent elimination. Tehran: Shayan Gostar Publication; 2018.

2. World Health Organization. World malaria report 2020: 20 years of global progress and challenges. Geneva: World Health Organization; 2020.

3. Vatandoost H, Raeisi A, Saghafipour A, Nikpour F, Nejati J. Malaria situation in Iran: 2002-2017. Malar J. 2019;18(1):1-7.

4. Hemami MR, Sari AA, Raeisi A, Vatandoost $\mathrm{H}$, Majdzadeh R. Malaria elimination in 
Iran, importance and challenges. Int J Prev Med. 2013;4(1):88-94.

5. SheikhzadehK,HaghdoostAA,Bahrampour A, Raeisi A, Zolala F, et al. Predicting malaria transmission risk in endemic areas of Iran: A multilevel modeling using climate and socioeconomic indicators. Iran Red Crescent Med J. 2017;19(4):e45132.

6. Kong X, Liu X, Tu H, Xu Y, Niu J, Wang Y, etal. Malaria control and prevention towards elimination: Data from an eleven-year surveillance in Shandong Province, China. Malar J. 2017;16(1):55.

7. Diallo MA, Badiane AS, Diongue K, Deme A, Lucchi NW, Gaye M, et al. Non-falciparum malaria in Dakar: A confirmed case of Plasmodium ovale wallikeri infection. Malar J. 2016;15(1):1-6.

8. World Health Organization. WHO malaria terminology. Geneva: World Health Organization; 2016.

9. UCSF Global Health Group. Eliminating malaria in Iran. Son Francisco: University of California; 2015.

10. Shahin A, Abdelbaky N, Emam S. A case of malignant malaria complicated by symmetrical peripheral gangrene. J Rheum Dis Treat. 2015;1:016. 